\title{
recithunds
}

Revista Científica Mundo de la Investigación y el Conocimiento

Alvaro Peralta Beltrán ${ }^{\text {a; }}$ Carlos Fernando Bombón Escobar ${ }^{\text {b; }}$ Francisco Xavier Hugo Cardenas ${ }^{c}$; Carlos Roberto Flores Ramos ${ }^{d}$

Impacto del Programa de Organización Saludable en las empresas financieras

Impact of the Healthy Organization Program on financial companies

Revista Científica Mundo de la Investigación y el Conocimiento. Vol. 3 núm.3. Esp., noviembre, ISSN: 2588-073X, 2019, pp. 725-744

DOI: 10.26820/recimundo/3.(3.Esp).noviembre.2019.725-744

URL: http://recimundo.com/index.php/es/article/view/632

Código UNESCO: 3212 Salud Publica

Tipo de Investigación: Artículo de Revisión

(C) RECIMUNDO; Editorial Saberes del Conocimiento, 2019

Recibido: $15 / 09 / 2019$

Aceptado: 23/10/2019

Publicado: 30/11/2019

Correspondencia: alvaroperalt@gmail.com

a. Magister en Seguridad, Salud y Ambiente; Doctor en Psicología Industrial; Investigador Independiente; Guayaquil, Ecuador; alvaroperalt@gmail.com

b. Magister en Seguridad, Salud y Ambiente; Ingeniero Industrial; Investigador Independiente; Guayaquil, Ecuador; carlosferbe1@hotmail.com

c. Magister en Seguridad, Salud y Ambiente; Ingeniero Ambiental; Investigador Independiente; Guayaquil, Ecuador; fcohugo@hotmail.com

d. Magister en Seguridad, Salud y Ambiente; Magister en Administración de Empresas con Especialidad en Gestión Integrada de Calidad, Seguridad y Medio Ambiente; Investigador Independiente; Guayaquil, Ecuador; crfrind@ hotmail.com 


\section{Impacto del Programa de Organización Saludable en las empresas financieras}

Vol. 3, núm. 3 Esp., (2019)

Alvaro Peralta Beltrán; Carlos Fernando Bombón Escobar; Francisco Xavier Hugo Cardenas; Carlos Roberto Flores Ramos

\section{RESUMEN}

El programa de organización saludable busca darle verdadera importancia al valor que tiene la salud en el ámbito del trabajo, demostrando a su vez, cómo este tipo de plan empresarial representa un valor estratégico en lo que refiere a la gestión y desarrollo de los recursos humanos. Entiéndase de esta forma que, la comprensión de organización saludable, refiere a un modelo integrador, el cual beneficia en primer lugar al capital o talento humano, siendo esto de gran importancia, puesto que es el mismo el encargado de desarrollar de manera efectiva las funciones empresariales y de esta forma, mejorar la productividad y estados financieros de las organizaciones. Entiéndase con esto que, las organizaciones saludables cuidan no solo de las relaciones entre los empleados y dirección dentro de la propia organización, sino también de las relaciones de la organización como un todo con su ambiente extra-organizacional, con la comunidad cercana y la sociedad en general. Uno de los objetivos de estas organizaciones es tener un impacto positivo en el ambiente local externo a la organización, así como ofrecer una imagen positiva y saludable de la empresa hacia el exterior; y ello lo consiguen mediante la cooperación entre partners en la cadena de producción y con el apoyo de otras empresas y organizaciones externas. Otro de los propósitos de este tipo de programas es la responsabilidad social de la empresa, también denominada responsabilidad social corporativa, entendida como un término que hace referencia al conjunto de obligaciones y compromisos, legales y éticos, tanto nacionales como internacionales, que se derivan de los impactos que las actividades de las organizaciones producen en el ámbito social, laboral, medioambiental y de los derechos humanos, beneficiando al conjunto organizacional en pleno.

Palabras Claves: Programa de organización Saludable; Empresas financieras; Sector Laboral; Talento Humano; Salud Laboral. 


\title{
Impacto del Programa de Organización Saludable en las empresas financieras
}

Vol. 3, núm. 3 Esp., (2019)

Alvaro Peralta Beltrán; Carlos Fernando Bombón Escobar; Francisco Xavier Hugo Cardenas;

Carlos Roberto Flores Ramos

\begin{abstract}
The healthy organization program seeks to give true importance to the value of health in the workplace, demonstrating, in turn, how this type of business plan represents a strategic value in terms of the management and development of human resources. Understand in this way that, the understanding of healthy organization, refers to an integrative model, which benefits in the first place to the capital or human talent, being this of great importance, since it is the same in charge of effectively developing the functions business and in this way, improve the productivity and financial statements of organizations. Understand with this that, healthy organizations take care not only of the relationships between employees and management within the organization itself, but also of the relationships of the organization as a whole with its extra-organizational environment, with the nearby community and society usually. One of the objectives of these organizations is to have a positive impact on the local environment external to the organization, as well as to offer a positive and healthy image of the company abroad; and this is achieved through cooperation between partners in the production chain and with the support of other companies and external organizations. Another purpose of this type of program is the company's social responsibility, also called corporate social responsibility, understood as a term that refers to the set of legal and ethical obligations and commitments, both national and international, that derive from the impacts that the activity of the organizations produce in the social, labor, environmental and human rights field, benefiting the entire organizational group.
\end{abstract}

Keywords: Healthy organization program; Financial companies; Labor Sector; Human Talent; Occupational Health. 


\section{Impacto del Programa de Organización Saludable en las empresas financieras}

Vol. 3, núm. 3 Esp., (2019)

Alvaro Peralta Beltrán; Carlos Fernando Bombón Escobar; Francisco Xavier Hugo Cardenas;

Carlos Roberto Flores Ramos

\section{Introducción.}

Desde hace ya un tiempo se viene escuchando el término «organización saludable» (Healthy organization). Parece un término novedoso y actual, que sin duda lo es, pero como ocurre con lo nuevo, ha generado también mucha confusión alrededor de su significado y alcance. Ahora bien, por otra parte, la investigación científica, con gran aplicación práctica, como es el caso del desarrollo de los recursos humanos, necesita de conceptos «nuevos» que capten la atención del management para dar un giro a las prácticas empresariales, con el objetivo de la mejora y renovación continua. (Cencott, 2016).

Por ende, es importante señalar que el sector empresarial de hoy día, se encuentra ante el calificativo de «saludable, cuando brinda a las organizaciones un matiz centrado en el «cuidado» de la salud tanto de los empleados, como del mismo contexto en su conjunto. (Cencott, 2016). Esta definición, parte del Modelo de Empresa Saludable de AENOR, el cual se ha transformado para adaptarse a la realidad de las organizaciones seguras, saludables y sostenibles. El mismo, se encuentra integrado la Estructura de Alto Nivel de las Normas Internacionales ISO, logrando con ello facilitar su integración con otros sistemas de gestión.

En efecto, este proceso de transformación ha coincidido con la publicación de la Norma ISO 45001 de gestión de la seguridad y salud en el trabajo, con la que el Modelo de Empresa Saludable está estrechamente relacionado. (Cencott, 2016). Su objetivo específico, se ha centrado en incluir un anexo que guiara a las organizaciones en el diseño, planificación, desarrollo, seguimiento y mejora continua del sistema de gestión, de los planes, de los programas 


\section{Impacto del Programa de Organización Saludable en las empresas financieras}

Vol. 3, núm. 3 Esp., (2019)

Alvaro Peralta Beltrán; Carlos Fernando Bombón Escobar; Francisco Xavier Hugo Cardenas;

Carlos Roberto Flores Ramos

y de las acciones que pongan en marcha para dar respuesta a sus necesidades, tomando en cuenta la idea de empresa saludable.

Se puede decir entonces que, el modelo AENOR ha realizado un proceso de transformación de empresa saludable, adaptando el mismo, a una tendencia cultural; su objetivo general, ha sido, por un lado, facilitar su integración con otros sistemas gestión gracias a la Estructura de Alto Nivel de ISO, lográndose con ello, minimizar los recursos necesarios, simplificando la información documentada necesaria. Y, por otro, ampliar el concepto de "empresa saludable" a cualquier tipo de organización y a sus partes interesadas

En términos generales, a través de este modelo se trata de implementar cambios que eran necesarios para dar respuesta a la realidad actual de las empresas. (Garrido, 2016). Este proceso de transformación ha coincidido con la publicación de la Norma ISO 45001 de gestión de la seguridad y salud en el trabajo, con la que el Modelo de Empresa Saludable debe estar estrechamente relacionado. En definitiva, en este nuevo modelo las organizaciones encontrarán una herramienta más fácil para su aplicación e integración, y de gran eficacia en la gestión de empresas seguras, saludables, sostenibles y comprometidas, independientemente de su tamaño, sector y ubicación geográfica.

Para el año 2013 se da a conocer el Modelo de Empresa Saludable de AENOR, a través del mismo se establece que es una excelente herramienta, la cual permite estructurar y dirigir actividades médico-preventiva, así como de promoción de la salud y el bienestar de los trabajadores y las trabajadoras y su entorno. (Garrido, 2016). La estructura del modelo, la amplitud de sus escenarios de trabajo y sus directrices permitían sistematizar la actividad y los 


\section{Impacto del Programa de Organización Saludable en las empresas financieras}

Vol. 3, núm. 3 Esp., (2019)

Alvaro Peralta Beltrán; Carlos Fernando Bombón Escobar; Francisco Xavier Hugo Cardenas; Carlos Roberto Flores Ramos

esfuerzos realizados empresariales desarrollados hasta entonces; a la vez que facilitaban muy considerablemente la planificación y el desarrollo de nuevos proyectos.

Por todo esto, se logra entender que el Modelo de Empresa Saludable pasó a ser una de las líneas fundamentales del Plan Director de Recursos Humanos en línea con el Plan Estratégico de la compañía, lo que demuestra el alto nivel de compromiso de la dirección con el bienestar de las personas de nuestra empresa. (Garrido, 2016). Todo esto, llevó a incorporar cada vez más acciones enfocadas a generar ambientes de trabajo saludables y a contribuir al bienestar en todos los ámbitos contemplados en el modelo, como la prevención y promoción de la salud con la implantación de hábitos de vida saludables; la mejora del entorno psicosocial, progresando en la conciliación de la vida laboral y personal en un entorno de trabajo flexible y en la gestión de las emociones.

Se logra entender entonces que, el Modelo de Empresa Saludable de AENOR tiene además un papel protagonista en el alineamiento con el Compromiso de Sostenibilidad, pues tiene como objetivo central mejorar el bienestar de las personas que conforman el capital humano de la empresa y lograr la adaptación a los nuevos tiempos, lo que supone una transformación cultural y la implantación de nuevas formas de trabajo, a las que sin duda también contribuye este modelo. (Garrido, 2016). De ahí, que la cultura de una organización segura, saludable y sostenible deberá evolucionar hasta alcanzar:

- La consecución de unos trabajadores, sus familias, clientes, usuarios, personas y otras partes interesadas más sanos, motivados y satisfechos que sientan con orgullo la pertenencia a dicha organización o tener relación con ella. 


\section{Impacto del Programa de Organización Saludable en las empresas financieras}

Vol. 3, núm. 3 Esp., (2019)

Alvaro Peralta Beltrán; Carlos Fernando Bombón Escobar; Francisco Xavier Hugo Cardenas; Carlos Roberto Flores Ramos

- Una mejora en todos los entornos bajo la capacidad de influencia de la organización, que la hagan atractiva para otros profesionales y que, además, retiene el talento.

- Un incremento de los clientes, usuarios y otras partes interesadas en el uso de los productos o servicios responsables que la organización ofrece.

- Una mejora de la imagen corporativa mediante la proyección de una organización segura, saludable y responsable en la que pueden participar los propios trabajadores, clientes, proveedores y otras partes interesadas hasta llegar a convertirse en un referente en su sector y dentro de la sociedad.

La Organización Mundial de la Salud, OMS, a través de su modelo de entornos saludables establece cuatro avenidas que son: ambiente físico de trabajo, entorno psicosocial de trabajo, recursos personales en el trabajo y participación de la empresa en la comunidad; todo, esto, tomando en cuenta la legislación existente sobre seguridad y salud laboral, la cual es tratada en todos los países, siendo las dos primeras ya manejadas por la gran mayoría de las organizaciones en el marco de sus sistemas de gestión de la seguridad y la salud laboral (SSL), implementándose para ello, el sistema de gestión Estándar OHSAS 18001 o, el actual concebido por la Norma ISO 45001. (Garrido, 2016).

De este modo, se logra precisar que es, a través del Modelo de Empresa Saludable de AENOR que las empresas logran unificar una sola área la gestión, la cual postula la: salud y seguridad de las personas de la organización. Dicho modelo, busca promocionar e implantar hábitos saludables en el ámbito de las personas, sus familias y el entorno, lo que lleva a definir esta área, como área de Estilo de vida, donde tendrá cabida todo tipo de acciones centradas en los 


\section{Impacto del Programa de Organización Saludable en las empresas financieras}

Vol. 3, núm. 3 Esp., (2019)

Alvaro Peralta Beltrán; Carlos Fernando Bombón Escobar; Francisco Xavier Hugo Cardenas; Carlos Roberto Flores Ramos

factores de riesgo relacionados con hábitos de vida y salud dentro y fuera de la organización. (Garrido, 2016).

Asimismo, se logra precisar que los elementos de este modelo que influirán en los entornos psicosociales laborales son, el estilo de gestión del trabajo de las personas, las políticas de conciliación de recursos humanos, la comunicación, el liderazgo y compromiso, el clima laboral, la cultura de la organización con respecto a las personas y su bienestar; contribuyendo todos estos elementos con la formación de una cultura positiva en seguridad, salud y bienestar laboral (SSBL). (Garrido, 2016). He allí, que muchas organizaciones cuentan con proyectos dentro del marco de su compromiso con la comunidad y se encuentran centrados en promover y gestionar acciones para la sociedad mediante acciones focalizadas en el cuidado del medio ambiente y del bienestar global de la misma.

Ejemplos de estas acciones, son los planes de acción enfocados a mejorar las posibilidades y oportunidades de la comunidad donde se establece la organización, al mantenimiento de la ética empresarial y lucha contra la corrupción, la contribución a la sociedad a través de productos y servicios útiles y en condiciones justas, o el respeto al medio ambiente evitando la contaminación, minimizando la gestión de residuos y racionalizando el uso de los recursos naturales y energéticos. (Garrido, 2016).

En términos generales, se observa la importancia que tiene el modelo del programa de empresa saludable, el cual se encuentra apoyado en una visión holística, que supone que la salud va a representar siempre un bien fundamental, el cual forma parte de la propia dignidad del ser 


\section{Impacto del Programa de Organización Saludable en las empresas financieras}

Vol. 3, núm. 3 Esp., (2019)

Alvaro Peralta Beltrán; Carlos Fernando Bombón Escobar; Francisco Xavier Hugo Cardenas; Carlos Roberto Flores Ramos

humano; siendo entonces, su adecuada prevención y protección viene siendo norma básica de todas las actividades y estrategias de gestión que logren implementarse a nivel empresarial.

\section{Metodología.}

\section{Fuentes documentales}

El análisis correspondiente a las características que predomina en el tema seleccionado, llevan a incluir diferentes fuentes documentales encargadas de darle el respectivo apoyo y en ese sentido cumplir con la valoración de los hechos a fin de generar nuevos criterios que sirven de referencia a otros procesos investigativos. Para (Arias, 2010), las fuentes documentales incorporadas en la investigación documental o bibliográfica, "representa la suma de materiales sistemáticos que son revisados en forma rigurosa y profunda para llegar a un análisis del fenómeno". (p.41). Por lo tanto, se procedió a cumplir con la realización de una lectura previa determinada por encontrar aquellos aspectos estrechamente vinculados con el "EL IMPACTO DEL PROGRAMA DE ORGANIZACIÓN SALUDABLE EN LAS EMPRESAS FINANCIERAS” para luego explicar mediante un desarrollo las respectivas apreciaciones generales de importancia.

Técnicas para la Recolección de la Información

La conducción de la investigación para ser realizada en función a las particularidades que determinan a los estudios documentales, tiene como fin el desarrollo de un conjunto de acciones encargadas de llevar a la selección de técnicas estrechamente vinculadas con las características 


\section{Impacto del Programa de Organización Saludable en las empresas financieras}

Vol. 3, núm. 3 Esp., (2019)

Alvaro Peralta Beltrán; Carlos Fernando Bombón Escobar; Francisco Xavier Hugo Cardenas; Carlos Roberto Flores Ramos

del estudio. En tal sentido, (Arias Ob cit) refiere, que es "una técnica particular para aportar ayuda a los procedimientos de selección de las ideas primarias y secundarias”. (p. 71).

Por ello, se procedió a la utilización del subrayado, resúmenes, fichaje, como parte básica para la revisión y selección de los documentos que presentan el contenido teórico. Es decir, que mediante su aplicación de estas técnicas se pudo llegar a recoger informaciones en cuanto a la revisión bibliográfica de los diversos elementos encargados de orientar el proceso de investigación. Tal como lo expresa, (Arias Ob cit) "las técnicas documentales proporcionan las herramientas esenciales y determinantes para responder a los objetivos formulados y llegar a resultados efectivos" (p. 58). Es decir, para responder con eficiencia a las necesidades investigativas, se introdujeron como técnica de recolección el método inductivo, que hizo posible llevar a cabo una valoración de los hechos de forma particular para llegar a la explicación desde una visión general.

Asimismo, se emplearon las técnicas de análisis de información para la realización de la investigación que fue ejecutada bajo la dinámica de aplicar diversos elementos encargados de determinar el camino a recorrer por el estudio, según, (Arias, Ob cit) las técnicas de procesamiento de datos en los estudios documentales "son las encargadas de ofrecer al investigador la visión o pasos que debe cumplir durante su ejercicio, cada una de ellas debe estar en correspondencia con el nivel a emplear" (p. 123). Esto indica que, para llevar a cabo el procesamiento de los datos obtenidos, es necesario establecer las técnicas que serán seleccionadas, destacándose en este caso, de manera particular: fichas de resumen, textual, registros descriptivos entre otros, los mismos se deben ajustar al nivel que ha sido seleccionado. 


\section{Impacto del Programa de Organización Saludable en las empresas financieras}

Vol. 3, núm. 3 Esp., (2019)

Alvaro Peralta Beltrán; Carlos Fernando Bombón Escobar; Francisco Xavier Hugo Cardenas;

Carlos Roberto Flores Ramos

\section{Resultados.}

\section{Organizaciones Saludables}

Las organizaciones saludables llevan implícitos dos conceptos: organización y salud. Por una parte, la organización hace referencia a las formas en que se estructuran y gestionan los procesos de trabajo, incluyendo el diseño de los puestos, los horarios de trabajo, el estilo de dirección, la efectividad organizacional y las estrategias organizacionales para la adaptación de los empleados, esto se traduce en las prácticas de desarrollo de los recursos humanos. Por ende, la adición del término saludable deriva de la idea de que es posible distinguir sistemas de organizaciones sanos y enfermos. Es decir, distinguir, en definitiva, formas de estructurar y gestionar los procesos de trabajo con resultados más saludables que otros.

Imagen 1. Programa de empresa Saludable

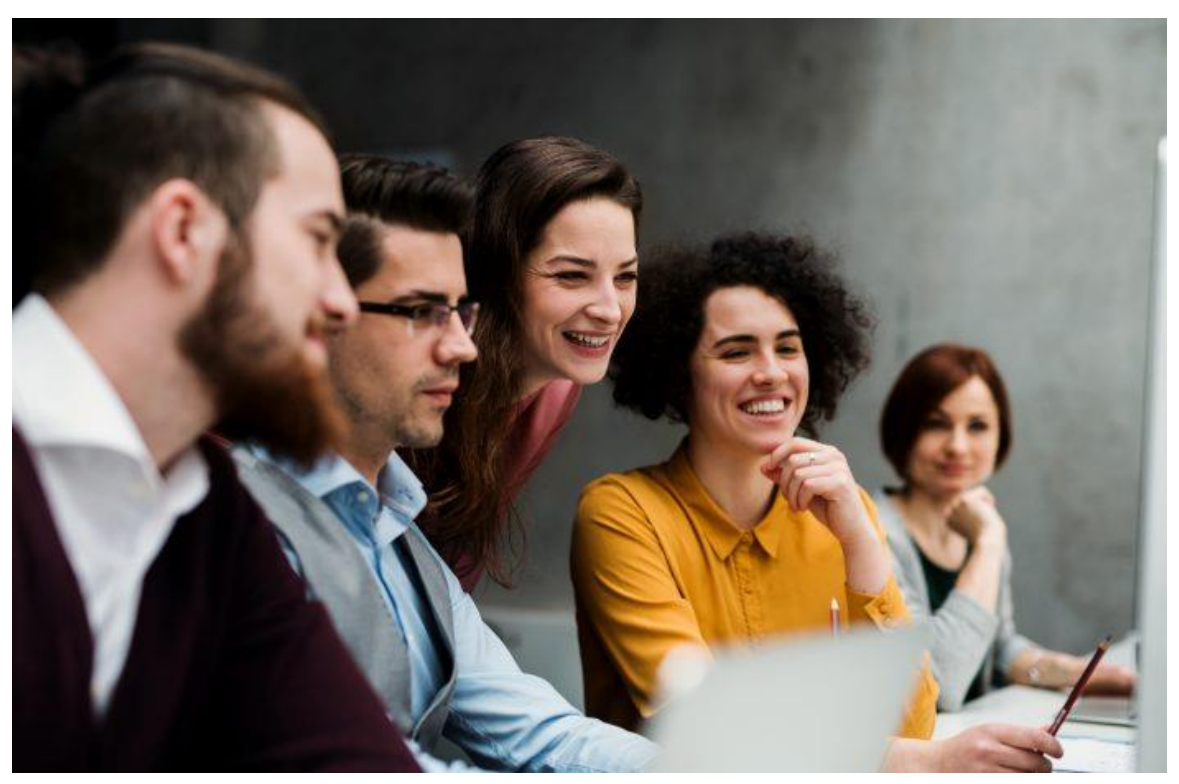

Fuente: (Garrido, 2016). 


\section{Impacto del Programa de Organización Saludable en las empresas financieras}

Vol. 3, núm. 3 Esp., (2019)

Alvaro Peralta Beltrán; Carlos Fernando Bombón Escobar; Francisco Xavier Hugo Cardenas; Carlos Roberto Flores Ramos

Este concepto de organización saludable, tiene implicaciones importantes que incluyen las relaciones trabajo-salud. A través de estas políticas se enfatiza en primer lugar en la estructura y la forma cómo es organizado el trabajo, el cual tiene un impacto directo en la salud y bienestar de los empleados. Segundo, combinar el término saludable con organización conlleva una visión integradora, incluyendo resultados de un variado número de disciplinas y especialidades, que comprende no solo las perspectivas tradicionales de salud en el lugar de trabajo como el estrés ocupacional, la seguridad y salud ocupacional y la promoción de la salud laboral, sino también la conducta organizacional, la dirección y la economía. (Garrido, 2016).

En consecuencia, se puede entender que la investigación sobre las organizaciones saludables representa la convergencia de la investigación científica realizada en distintas disciplinas el concepto de organización saludable imprime a la salud en el trabajo de una orientación hacia el capital humano y social, y el más actual, así llamado: capital psicológico positivo y conecta la optimización del capital humano, social y psicológico, con la maximización de la estrategia de negocio. (Cuencas, 2015). Además, existen beneficios colaterales en el hecho de que las organizaciones saludables deberían ser capaces de atraer y retener trabajadores más productivos y talentos, y a la vez, tener mayor éxito en la gestión de costes siendo también más competitivas 


\section{Impacto del Programa de Organización Saludable en las empresas financieras}

Vol. 3, núm. 3 Esp., (2019)

Alvaro Peralta Beltrán; Carlos Fernando Bombón Escobar; Francisco Xavier Hugo Cardenas;

Carlos Roberto Flores Ramos

Figura 1. Elementos de un programa de Organización Saludable

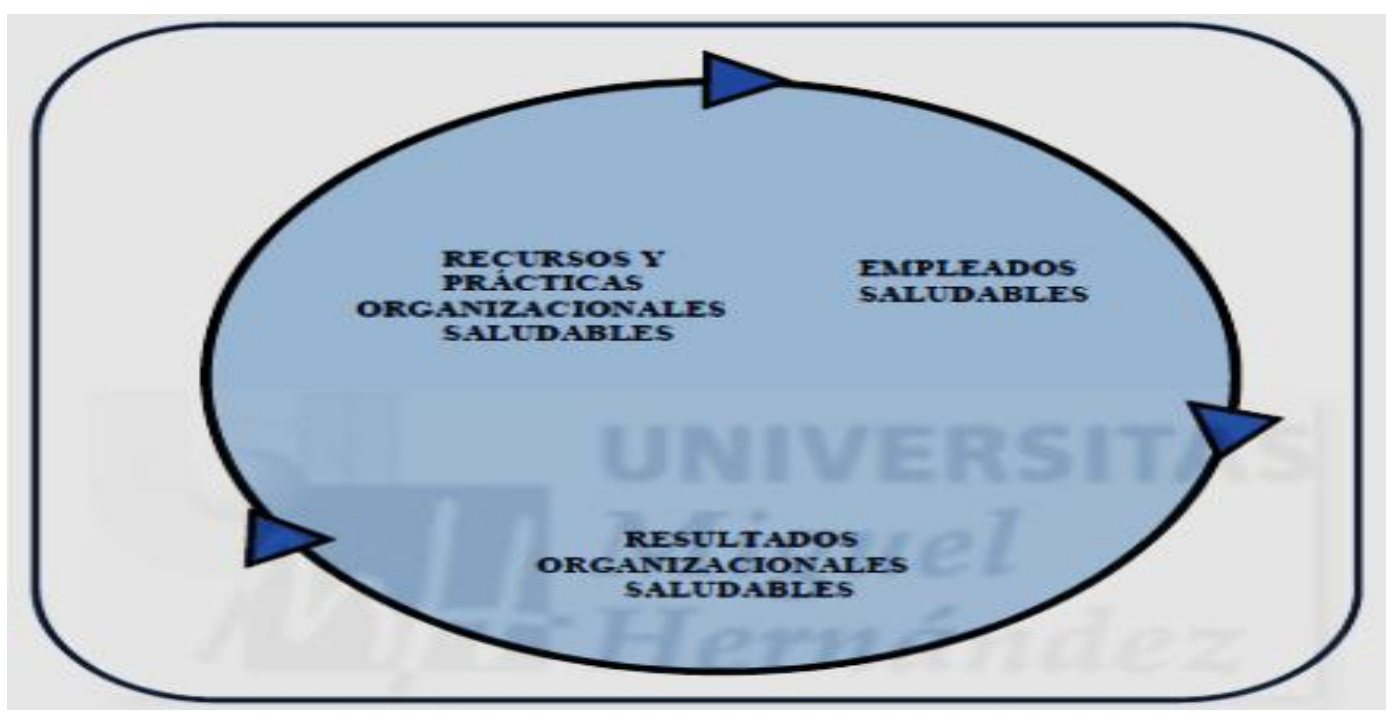

Fuente: (Cuencas, 2015).

De manera puntual, se establece que las organizaciones saludables cuidan no solo de las relaciones entre los empleados y dirección dentro de la propia organización, sino también de las relaciones de la organización como un todo con su ambiente extra-organizacional, con la comunidad cercana y la sociedad en general. Uno de los objetivos de estas organizaciones es tener un impacto positivo en el ambiente local externo a la organización, así como ofrecer una imagen positiva y saludable de la empresa hacia el exterior; y ello lo consiguen mediante la cooperación entre partners en la cadena de producción y con el apoyo de otras empresas y organizaciones externas. (Cuencas, 2015).

Uno de los temas que caracteriza este tipo de programas es la responsabilidad social de la empresa, también denominada responsabilidad social corporativa. Es un término que hace referencia al conjunto de obligaciones y compromisos, legales y éticos, tanto nacionales como 


\section{Impacto del Programa de Organización Saludable en las empresas financieras}

Vol. 3, núm. 3 Esp., (2019)

Alvaro Peralta Beltrán; Carlos Fernando Bombón Escobar; Francisco Xavier Hugo Cardenas;

Carlos Roberto Flores Ramos

internacionales, que se derivan de los impactos que las actividades de las organizaciones producen en el ámbito social, laboral, medioambiental y de los derechos humanos. (Cuencas, 2015).

Figura 2. Estructura de un programa de Organización Saludable

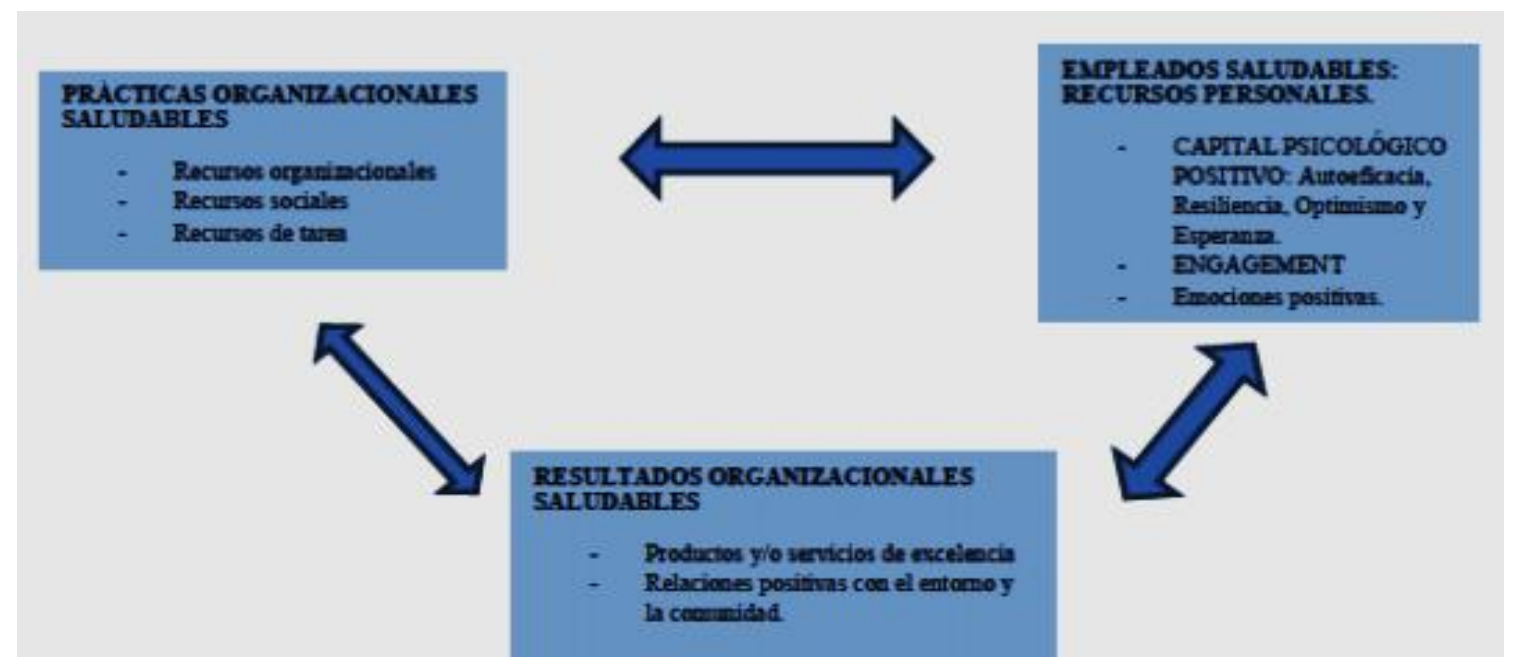

Fuente: (Cuencas, 2015).

Hoy en día las empresas son cada vez más conscientes de la necesidad de incorporar las preocupaciones sociales. Ambos conceptos, organización saludable y responsabilidad social corporativa tienen en cuenta conceptos e ideas afines como la necesidad de desarrollar empleados saludables (las personas) en un ambiente sano (el planeta), y en un sentido metafórico de la palabra, una organización saludable es una organización con éxito financiero. (Cuencas, 2015). No obstante, el mecanismo para alcanzar estos resultados organizacionales saludables se basa en la gestión del «capital psicológico positivo, el cual hace referencia a la existencia de 


\section{Impacto del Programa de Organización Saludable en las empresas financieras}

Vol. 3, núm. 3 Esp., (2019)

Alvaro Peralta Beltrán; Carlos Fernando Bombón Escobar; Francisco Xavier Hugo Cardenas; Carlos Roberto Flores Ramos

«salud» entre los empleados de la organización, entendida como un estado de completo bienestar, pero no solo físico, sino también social y psicológico.

Impacto del Programa de Organización Saludable en las empresas Financieras

El tema central en el estudio de las organizaciones saludables es precisamente la «salud» de los empleados, pero también el buen funcionamiento de la organización en cuanto a sus beneficios económicos y sociales. Desde una perspectiva más positiva se pueden estudiar las organizaciones saludables optimizando el capital humano, el capital social y el así llamado capital psicológico, a la vez que maximizamos la estrategia de negocio. (Cuencas, 2015). Por este motivo es que en este artículo se tratará el estudio de las organizaciones saludables desde una perspectiva diversa en cuanto a las disciplinas científicas de base (psicología ocupacional, management, conducta organizacional, etc.), pero se acentuará la investigación derivada de la Psicología Positiva que imprime un carácter más positivo al estudio científico del fenómeno.

Por ende, se debe tener en cuenta que una de las razones de imprimir este carácter más positivo tiene que ver con el hecho de que para poder sobrevivir y prosperar en un contexto de cambio económico y social continuo, las organizaciones necesitan tener empleados motivados y psicológicamente sanos, y que para poder conseguirlo las políticas de recursos humanos deben estar sincronizadas. (Cuencas, 2015). La razón es que las organizaciones modernas están cambiando en una dirección que se basa cada vez más en el conocimiento psicológico y la experiencia, en la autogestión y la atención a necesidades individuales y colectivas de los empleados, de la organización y de la sociedad en general. 


\section{Impacto del Programa de Organización Saludable en las empresas financieras}

Vol. 3, núm. 3 Esp., (2019)

Alvaro Peralta Beltrán; Carlos Fernando Bombón Escobar; Francisco Xavier Hugo Cardenas; Carlos Roberto Flores Ramos

En concreto, en el ámbito de la gestión de la salud en el trabajo y las organizaciones, este paradigma tradicional se ha centrado en el lado negativo o el lado «oscuro» del estrés laboral, inspirado en el modelo médico. Pero solo cubre la mitad del espectro del fenómeno. Por tanto, existe una necesidad creciente de una aproximación positiva, más novedosa y emergente que se centre en las fortalezas del empleado y del funcionamiento organizacional óptimo, que ofrezca respuestas a demandas emergentes en cuanto a los cambios que están aconteciendo en las sociedades modernas. (Cuencas, 2015).

Estos cambios organizacionales, a su vez, impactan en los puestos de trabajo e influyen en la seguridad, la. salud y el bienestar de los empleados. Si no se gestionan bien estos cambios, a la larga puede llevar a la aparición de organizaciones «enfermas» que se caracterizan por su falta de efectividad e inadaptación al entorno. De ahí, que las organizaciones modernas esperan que sus empleados sean proactivos y muestren iniciativa personal, que colaboren con los demás, que sean responsables en su propio desarrollo de carrera y que se comprometan con la «excelencia» empresarial. Este objetivo empresarial no puede alcanzarse con una fuerza laboral «saludable» al estilo tradicional: empleados satisfechos con sus trabajos, que no experimentan estrés laboral y que muestran bajos índices de absentismo. (Cuencas, 2015).

Se necesita algo más para poner en marcha toda la maquinaria organizacional y conseguir este objetivo. Los modelos de gestión tradicionales que consideran a los empleados como instrumentos para conseguir los fines empresariales, están siendo reemplazados por una genuina Psicología Ocupacional u Organización Positiva (PoP) que considera la salud del empleado como una meta en sí misma y un objeivo legítimo que debe incluirse en las políticas 


\section{Impacto del Programa de Organización Saludable en las empresas financieras}

Vol. 3, núm. 3 Esp., (2019)

Alvaro Peralta Beltrán; Carlos Fernando Bombón Escobar; Francisco Xavier Hugo Cardenas;

Carlos Roberto Flores Ramos

organizacionales. El objetivo es, pues, describir, explicar y predecir el funcionamiento óptimo en estos contextos, así como optimizar y potenciar la calidad de vida laboral y organizacional.

Factores de Éxito

Implantar el Modelo de Empresa Saludable de AENOR debe partir de una decisión estratégica y de un imperativo ético de la organización; esta es la base de todo. El éxito de este sistema de gestión depende tanto del liderazgo de todas las partes implicadas, como de los compromisos que se asuman, desde los más individuales a los más colectivos y sociales. Por ello, la participación de todos los niveles y funciones dentro de la organización, por un lado, y de las partes interesadas externas a la organización por otro, es clave. (Cuencas, 2015).

Solo de este modo se podrá establecer una cultura del bienestar completa en la organización integrando, no solo la protección de la salud, sino la prevención y la promoción, no quedándose en el trabajador y en la empresa, sino extendiéndola a la persona, a su familia y al entorno donde vive. Solo partiendo del compromiso sincero de los líderes de la empresa, podrá integrarse esta cultura del bienestar en los procesos, en la estructura y en la gestión; en definitiva, en la estrategia de toda la organización. Para ello es fundamental que se designen los recursos necesarios y suficientes.

De acuerdo con este compromiso la organización definirá unos objetivos alineados con los objetivos clave de la empresa, que serán entendidos por todos sus integrantes. Este es el modo de implementar un sistema de gestión sostenible y continuado en el tiempo, en el que de manera natural se integra la participación, la consulta y la comunicación a los trabajadores, representantes y partes interesadas. La implicación de todos ellos permitirá lograr una cultura 


\section{Impacto del Programa de Organización Saludable en las empresas financieras}

Vol. 3, núm. 3 Esp., (2019)

Alvaro Peralta Beltrán; Carlos Fernando Bombón Escobar; Francisco Xavier Hugo Cardenas; Carlos Roberto Flores Ramos

colectiva del bienestar e inculcar de manera espontánea la responsabilidad de hacerla extensiva a su entorno familiar y personal, comunitario y a otras partes interesadas, multiplicándose de modo exponencial los efectos beneficiosos. (Cuencas, 2015).

Pero para que estos planes y programas de bienestar sean realmente beneficiosos, deben partir de una correcta identificación, evaluación y determinación de factores de riesgo relacionados con la salud, la seguridad de los integrantes de la organización, el estilo de vida, la responsabilidad de la empresa con su entorno y la cultura del bienestar organizacional. Todo ello sin olvidar los requisitos legales de seguridad y salud en el trabajo, que deben ser prioritarios. El resultado serán unos planes y programas de bienestar y compromiso social integrados en la estrategia global de la organización con la capacidad para romper con los "silos” y lograr los resultados previstos. (Cuencas, 2015).

Ahora bien, nada de esto tendría sentido si no se realizan esfuerzos para fomentar e incrementar la capacidad individual de cada persona con el fin de mantener un óptimo estado de salud y una calidad de vida adecuada en cada momento. Y tampoco si no se realiza la evaluación continua el desempeño del sistema para asegurar el logro de los objetivos marcados, la obtención de los resultados previstos y, en el caso de no ser así, tener el compromiso de realizar el análisis de los fallos que nos ayuden a generar nuevas oportunidades de mejora.

\section{Conclusiones.}

Las organizaciones saludables son definidas como «aquellas organizaciones caracterizadas por invertir esfuerzos de colaboración, sistemáticos e intencionales, los cuales permiten maximizar el bienestar de los empleados y la productividad, mediante la generación de 


\section{Impacto del Programa de Organización Saludable en las empresas financieras}

Vol. 3, núm. 3 Esp., (2019)

Alvaro Peralta Beltrán; Carlos Fernando Bombón Escobar; Francisco Xavier Hugo Cardenas; Carlos Roberto Flores Ramos

puestos bien diseñados y significativos, de ambientes sociales de apoyo, y finalmente mediante las oportunidades equitativas y accesibles para el desarrollo de la carrera y del balance trabajovida privada.

Una de las novedades es que este modelo ha evolucionado para integrar la Estructura de Alto Nivel de la Norma Internacional ISO incluida en las normas de sistemas de gestión. Así, se deberán analizar las oportunidades y no solo los riesgos, reforzando el carácter proactivo del modelo, que promueve actuar antes de que suceda el problema de seguridad, de salud, organizacional o en la comunidad.

Conforme a este programa, todas las organizaciones desarrollan un plan de implantación que permite garantizar la eficacia en el funcionamiento del sistema de gestión, el cual es primordial en el modelo para las personas con funciones y responsabilidades en él. Ello facilitará la identificación de los cambios necesarios y la puesta en marcha de estos dentro de la organización. Por último, las organizaciones deben verificar la implantación efectiva de los cambios de gestión mediante auditorías internas u otras herramientas.

Se puede decir entonces que el impacto generado por este programa en las organizaciones financieras se encuentra relacionado con la excelencia organizacional y el hecho de mantener relaciones positivas con el entorno organizacional, lo cual se ve demostrado, a través de los esfuerzos demostrado, a través de prácticas relacionadas con la promoción y optimización de una seríe de recursos relacionados con la mejora de las tareas (ej. diseño y rediseño de puestos), el ambiente social de la organización (ej. canales de comunicación abierta, estilos de liderazgo 


\section{Impacto del Programa de Organización Saludable en las empresas financieras}

Vol. 3, núm. 3 Esp., (2019)

Alvaro Peralta Beltrán; Carlos Fernando Bombón Escobar; Francisco Xavier Hugo Cardenas;

Carlos Roberto Flores Ramos

transparente) y la organización (ej. selección y socialización laboral, formación y desarrollo, políticas de estabilidad en el empleo, estrategias de conciliación trabajo/vida privada).

\section{Bibliografía.}

Arias, F. (2010). Paradigmas de la Investigación Científica. España: Editorial: Luces.

Cencott, P. (2016). Programa de Organización Saludable. Cuaderno de desarrollo económico, 3, $12-22$.

Cuencas, E. (2015). Programas de Organización Saludable. Revista Vida Económica de las Organizaciones de la Universidad Nacional de México, 1-24.

Garrido, A. (2016). Efectos de los Programas de Organización Saludable en empresas. Foro Regional sobre Política Industrial, 34-38.

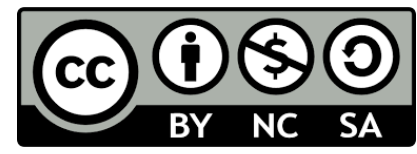

RECONOCIMIENTO-NOCOMERCIAL-COMPARTIRIGUAL

CC BY-NC-SA

ESTA LICENCIA PERMITE A OTROS ENTREMEZCLAR, AJUSTAR Y CONSTRUIR A PARTIR DE SU OBRA CON FINES NO

COMERCIALES, SIEMPRE Y CUANDO LE RECONOZCAN LA AUTORÍA Y SUS NUEVAS CREACIONES ESTÉN BAJO UNA LICENCIA CON LOS MISMOS TÉRMINOS. 